\title{
TEMPERATURE VARIATION AND SPECIES INTERACTIONS IN AQUATIC SYSTEMS in Grand Teton National PaRk
}

\author{
BRUCE WOODWARD $\bullet$ REYNOLDS ELECTRICAL AND \\ ENGINEERING CO., INC. LAS VEGAS \\ SANDRA MITCHELL • DEPARTMENT OF BIOLOGY \\ WESTERN WYOMING COLLEGE ^ ROCK SPRINGS
}

\section{$\checkmark$ ABSTRACT}

Temperature variation and species interactions were examined in small montane ponds in Grand Teton National Park during summer 1991. Temperature variation was pronounced, both over time and from place to place within one pond or across ponds. The magnitude of the temperature variation is such that it is likely to lead to large differences in the growth rates of poikilotherms that occupy these ponds.

Growth rates determine body sizes at any one point in time. Tadpole body size influenced the probability of predation by salamander larvae in these montane ponds in Grand Teton National Park. Given the natural body size ratios of predator and prey (observed in 1991), salamanders biased predation towards smaller tadpoles. Other predation experiments revealed that the salamander larvae biased predation towards tadpoles and away from damselfly naiads. This suggests that damselflies may experience lower predation by salamanders when in the presence of tadpoles than when in the absence of tadpoles.

Several species, including snails, caddisfly larvae, and tadpoles were very abundant in some ponds, but could be essentially absent from others. These three taxa appear to share similar diets. A simple experiment revealed the Pseudacris tadpoles and the snails compete, and that presence of salamander larvae had no effect on the strength of their competitive interaction

\section{$\checkmark \quad$ INTRODUCTION}

In 1991 we made several trips to Grand Teton National Park in the vicinity of the University of Wyoming field station. Our long term goal was to perform research that examines the effect of temperature variation on growth rate, and how differences in body sizes (an outcome of different growth rates) could influence species interactions. Because we had never worked in this area before, our immediate goals were to determine the range of temperature variation, and see what sorts of species interactions were taking place. In addition, we wanted to perform some experiments to determine if body size variation could influence species interactions, as it does in some other systems (Woodward 1983a,b, 1987, Woodward and Mitchell 1991).

Northern Wyoming is a mountainous area which contains many small shallow ponds. Montane ponds are commonly filled by snow melt, which means that the snowpack depth, and the temperatures in the early spring can have large effects on both pond depth, and on pond temperature. Most of the species inhabiting these small ponds are 
poikilotherms, and as such, have growth rates that are strongly influenced by temperature. This appeared to be a good place to perform this study.

\section{$\checkmark \quad$ METHODS}

To document which species co-occurred and the range of temperature variation, we visited Jackson Lake, small ponds near the field station, and small ponds along Pacific Creek Road. We were especially interested in working with the Northern Chorus Frog (Pseudacris triseriata), so we drove around at night listening for breeding choruses of this frog. We visited each pond at least once during the day, and most ponds were visited two or three times. We estimated pond surface area, made several depth readings to obtain an average, recorded water temperature within $2 \mathrm{~cm}$ of the pond surface, and made quantitative dip net surveys in each pond. In these surveys we made repeated sweeps through the water column and recorded the number of individuals per species per sweep. On some occasions we made temperature readings in the shade and sun, or at various depths, to get a feel for spatial temperature variation in a pond. We visited ponds in May, June and July.

Numerous organisms were collected and taken back to the boat house at the field station for identification, or to be used in predator-prey experiments. Three types of predation experiments were performed. In the simplest, we simply placed one predator and one potential prey together in a relatively small container, to see if the predator did prey on this particular species. We replicated this and all other experiments, as often as our supply of predators and potential prey would allow. No predator or prey was used more than once in any one experiment. This type of experiment was performed only if we had no knowledge of the predator-prey relationship of the species under study.

For most taxa we had some prior experience, and thus we performed one of two more interesting experiments. In the first, we offered the predator a choice between two size classes of its prey. The question to be answered here was whether prey size influenced the probability of a prey being eaten. A larger prey yields more energy if consumed, but may require more energy to capture and consume. Most aquatic predators are size-limited predators, which means that the prey can get too large to consume, and that over a wide range of prey sizes, increases in size lead to increased problems in prey handling for the predator. Many aquatic predators (e.g., fish, salamander larvae) ingest their prey whole, others (many insects) either suck the juices from the prey, or slowly break the prey into parts. Many of the insects take prey that are close to their own body size and typically outweigh them. For these species, increases in prey size makes it very difficult for the predator to hang onto the prey. The final type of predation experiment consisted of offering one predator a choice between two prey species. The question here was whether predators biased predation towards one or the other species. This question is important in its own right, and also has ramifications for questions related to indirect effects in the aquatic community (Morin 1988).

In each of our three types of predation experiment, one predator was exposed to either one or two potential prey items. The experiments took place in 1 - $6 \mathrm{~L}$ of pond water (container size depended on the size of predators and prey). There were no prey refuges in the experiments. Prey were scored as being eaten, killed, or left alone. For our questions, being killed was considered no different from being eaten. For the two types of predatorchoice experiments, we used a Sign test to determine if predation was biased towards either option to which the predator had been exposed.

Preliminary observations revealed that several species fed in the same manner and in the same areas, implying similar diets. To determine if these species might compete, we performed some simple competition experiments in $0.5 \times 0.5 \times 0.5 \mathrm{~m}$ screen mesh field cages. We collected caddisfly larvae and Psuedacris tadpoles from natural ponds. The caddisfly were separated into size classes, and we used the smallest body size class we could obtain (12-15 $\mathrm{mm}$ body length). Tadpoles were relatively small (5 - $8 \mathrm{~mm}$ body length). When we collected the caddisflies and the tadpoles we made quantitative sweeps to estimate densities/L. We used these natural densities in our experiment when we added 10 caddisfly larvae, or 15 tadpoles per cage. In this experiment we had three treatments: tadpoles only, caddisfly larvae only, or tadpoles and caddisfly larvae. Cages were placed in 30 to $35 \mathrm{~cm}$ of water 
in a natural pond located approximately $1 \mathrm{~km}$ east of the field station and west of state highway 191. Treatments were randomly assigned to field cages.

We also did another experiment to examine species interactions. In this experiment we tested for competitive interactions between snails and Pseudacris tadpoles, predation of salamander larvae on snails or tadpoles, and effects of predation by salamanders on any competitive interactions between snails and tadpoles. In this experiment, we had $\mathbf{3 0}$ field cages. Ten cages contained tadpoles only, ten contained tadpoles and snails, and ten contained snails only. Of these 30 cages, 15 also had two salamander larvae (44 - $48 \mathrm{~mm}$ total length) added per cage. Ten tadpoles or ten snails (these were natural densities) were added per cage. Treatments were randomly assigned to cages. The experiment was started on 6 July 1991 and ended on 24 July when all organisms were removed from the cages, towel dried to remove surface moisture, and weighed. Data analyses consisted of using two-way ANOVAs to compare mean weights, or number surviving per species per cage between the treatments of being raised alone or with a potential competitor, and being either in the presence or absence of salamander larvae.

\section{- RESULTS}

Water level on Jackson Lake raises at least $2 \mathrm{~m}$ in the spring time. The bottom in the vicinity of the field station consists of large rounded gravel or rocks. Finally, the wave action, due either to boat wakes or the wind, is strong and fairly consistent. Taken together these factors mean that the shallow water zone near the field station is not a very favorable place to live. I sampled for $\mathbf{3 0}$ minutes one day with a dip net and did not collect any macroinvertebrates (for comparison it is unusual to make two consecutive sweeps in the local small ponds and not collect anything). We gave up on Jackson Lake and concentrated our efforts at small ponds at Grand Teton National Park.

Temperature variation was pronounced at these small ponds in May, June and July. Simultaneous temperature readings could vary as much as 5 degrees $\mathrm{C}$ across a single pond. This variation is a function of sun versus shady areas, or springs versus nonspring areas. Temperatures differed by up to 10 degrees $\mathrm{C}$ across two ponds. This variation is probably due to sun versus shade, springs versus nonsprings, pond depth (shallow ponds heat up quicker in the spring), and early on, the degree to which snow melt contributes to the filling of the pond. Finally water temperature at the surface could vary as much as $\mathbf{5}$ degrees $\mathbf{C}$ across the day. Average temperatures were around 10 degrees $\mathrm{C}$ early in the growth season, later 20 degrees $\mathrm{C}$, thus site to site variation in temperature was very large. These sizeable differences in temperature could lead to large differences (probably on the order of $100 \%$ ) in growth rate.

The ponds shared many species in common. Tiger salamander larvae and dyticid beetle larvae were the only predators to be present in any numbers. Notonectids, dragonfly naiads, and hydrophilid beetle larvae were other predators that were present in small numbers, and werè large enough to eat the tadpoles, and most macroinvertebrates. Damselfly larvae were abundant in some ponds and can prey on the small macroinvertebrates (fly larvae). Fly larvae, corixids, tadpoles, caddisfly larvae, damselfly naiads (larvae), and snails were the predominant prey base in the system. Fairy shrimp were an important prey resource in a few ponds. As in other small pond systems we have studied, tadpoles, fairy shrimp, and snails can comprise very major components of the animal biomass in the water column. It is not uncommon for any one of these groups to comprise $>75 \%$ of the animal biomass in the water column (this excludes the difficult to sample burrowers). In several ponds, caddisfly larvae (this appears to be a single species) were also a major component of the animal biomass, approaching the mass of the snails or tadpoles.

We largely restricted our attention to the large and abundant predators (salamander larvae, dyticid beetle larvae, dragonfly naiads), and the potential prey that were very abundant (tadpoles, caddisfly larvae, and snails). These species varied a great deal in abundance from pond to pond. With our meager sampling effort we could document differences of an order of magnitude - we suspect that true differences probably approach two orders of magnitude. With the exception of fairy shrimp which were restricted to just a few ponds, the rest of these species appeared to be present in almost all ponds. Why the species were rare in some ponds and the major components of animal biomass in others is not clear. The tadpoles, snails, and caddisfly larvae are raspers that feed on periphyton attached to submerged 
objects. They feed in the same areas and probably have similar diets.

Predator-prey, or competitive interactions are likely explanations for the observed 10 fold pond to pond fluctuations of abundance of some of the species in this system. Whether body sizes of the interactants influences the outcome of these interactions, and thus contributes to the observed patterns of abundance, is not clear. We did perform a few experiments that shed some light on these questions.

Dyticid beetle larvae, dragonfly naiads, and salamander larvae were abundant enough that we collected individuals to run predation experiments. The beetle larvae were large relative to the prey items we offered them. We ran three experiments offering these larvae a choice between small and medium Pseudacris tadpoles, small and large tadpoles, and a choice between medium tadpoles and damselfly naiads. The dyticids exhibited no preference in any of the tests (Table 1). The dragonfly naiads were small relative to the size of their potential prey. We offered them a choice between small and medium tadpoles, and between small tadpoles and damselfly naiads. These predators did not eat any of the potential prey items in this experiment (Table 1). This was probably a function of their small size relative to the prey, as they do eat both of these prey species. Finally, we offered the salamanders a choice between small and medium tadpoles or between small tadpoles and damselfly naiads. The salamanders biased predation towards the smaller tadpoles relative to the medium sized ones, and were more likely to eat the tadpoles rather than the damselfly naiads (Table 1).

The caddisfly $\mathrm{x}$ tadpole experiment yielded no results because the water level dropped too much between two of our visits and left the cages stranded on land. The tadpole $x$ snail $x$ salamander (predation/competition experiment was moved to deeper water twice, and was more successful. The experiment was ended on 24 July before all tadpoles had transformed into little froglets. This early end to the experiment was dictated by our time budget, and leads to complications when interpreting the results for the frogs. We discuss these effects after stating the simple results.

The presence of the tadpoles had no effect on snail survival $(8.7+0.4$, mean + S.E., individuals per cage in presence of tadpoles, $8.7+$ 0.5 in absence of tadpoles, $F=0.001,1,17$ d.f., $p$ $=0.975$ ). Snail weight in the presence of tadpoles $(31.6+0.9 \mathrm{mg})$ was, however, $17 \%$ lower $(\mathrm{F}=$ $14.5,1,17$ d.f., $p<0.001)$ than snail weight in the absence of tadpoles $(38.0+1.4)$. Presence or absence of salamanders had no direct effect on snail survival $(F=0.39,1,17$ d.f, $p=0.54)$ or on snail wt $(F+0.006,1,17$ d.f., $p=0.81)$. These salamander larvae were probably too small to eat the snails. Adult salamanders in the ponds were gorged with snails. Finally, presence or absence of the salamanders had no effect on the influence of the tadpoles on the snails (i.e., the interaction term was nonsignificant).

There is some evidence that presence of the snails also influenced the frogs. Some tadpoles had transformed into froglets when we ended the experiment, so we analyze tadpole and froglet weights and survival as well as total number of frog offspring surviving. There is some evidence that presence of the snails may reduce tadpole survival $(1.6+0.5$ individuals per cage $)$, relative to when the snails are absent $(3.4+0.8, F=3.52,1,18$ d.f., $p+0.077)$. Presence of the snail had no effect on number of froglets surviving (snails present $3.2+$ 0.5 , snails absent $2.10 .6, F=1.91,1,18$ d.f., $p=$ 0.18 ), or total number of frog offspring surviving (snails present $4.8+0.6$, snails absent $5.5+1.0, \mathrm{~F}$ $=0.31,1,18$ d.f., $p=0.58)$. Presence of the snails had no effect on tadpole weight (snails present $28.2+2.0$, snails absent $28.5+2.6, F=0.08,1$, 13 d.f., $p=0.78$ ). Presence of the snails did lower froglet weight $23 \%$, (snails present $20.7+1.0$, snails absent $27.0+2.6, F=7.14,1,11$ d.f., $p=$ 0.022). Presence of the salamanders had no effect on tadpole survival $(F=1.5,1,18$ d.f., $p=0.24)$, number of froglets surviving $(F=0.36,1,18$ d.f., $p=0.56)$, total number of offspring surviving ( $F=$ $0.31,1,18$ d.f., $p=0.58)$, tadpole weight $(F=$ 1.73, 1, 13 d.f., $p=0.211$ ), and froglet weight ( $F$ $=1.3,1,11$ d.f., $p=0.28$ ). Again presence of the predator had no effect on any of the underlying competitive interactions.

Two cautions about the above results are in order. The first is that sample sizes are quite small and thus many sizeable differences in growth or survival are not regarded as significant. Should similar patterns hold with larger sample sizes many more results would have been statistically significant. The second caution relates to the results for the effect 
Table 1. Results of predation experiments performed at Grand Teton National Park in 1991. TL = total length, $\mathrm{BL}=$ body length exclusive of tail.

Predator (body size)

No. of prey consumed (body size)

Statistics

Dytcid beetle larvae

$(29-48 \mathrm{~mm} T L)$

Dytcid beetle larvae

(29 - $48 \mathrm{~mm}$ TL)

Dytcid beetle larvae

$(29-48 \mathrm{~mm} T L)$

Dragonfly naiad

(8-13 mm TL)

Dragonfly naiad

$(8-13 \mathrm{~mm}$ TL)

Salamander larvae

(34 - $58 \mathrm{~mm}$ TL)

Salamander larvae

(34 - $58 \mathrm{~mm}$ TL)
8 small tadpoles $(4.5$ - $8 \mathrm{~mm} \mathrm{BL})$

$N=13, x=5, p=0.58$

5 medium tadpoles (10 - $13 \mathrm{~mm} \mathrm{BL)}$

6 small tadpoles $(4.5-6.5 \mathrm{~mm} \mathrm{BL})$

$N=12, x=6, p=0.99$

6 large tadpoles (12 - $16 \mathrm{~mm} \mathrm{BL)}$

5 damselfly naiads (17 - $19 \mathrm{~mm}$ TL)

$\mathrm{N}=10, \mathrm{x}=5, \mathrm{p}=0.99$

5 tadpoles (11 - $12 \mathrm{~mm} \mathrm{BL)}$

0 small tadpoles (7 - $8.5 \mathrm{~mm} \mathrm{BL})$

no test

0 medium tadpoles (11 - $12 \mathrm{~mm} \mathrm{BL}$ )

0 small tadpoles (6 - $10.5 \mathrm{~mm} \mathrm{BL})$

no test

13 small tadpoles ( $5-8.5 \mathrm{~mm} \mathrm{BL}$ )

$\mathrm{N}=14, \mathrm{x}=1, \mathrm{p}=0.002$

1 medium tadpole (10 $13 \mathrm{~mm} \mathrm{BL}$ )

10 small tadpoles ( $6-8.5 \mathrm{~mm} \mathrm{BL}$ )

$\mathrm{N}=12, \mathrm{x}=2, \mathrm{p}=0.038$

2 damselfly naiads (16 - $19 \mathrm{~mm}$ TL) of the snails on the frogs. The tadpoles lose weight just before transforming into froglets. Because we ended the experiment just as tadpoles were starting to transform, this means that many tadpoles were at a stage where a low body weight could imply either lack of food (evidence of competition), or more rapid growth (a beneficial trait for species occupying drying ponds). Thus interpreting differences in tadpole weight is problematic for this data set. We suggest that the results for the frogs be regarded as suggestive rather than definitive.

\section{DISCUSSION}

Our results reveal significant temperature variation in small ponds in Grand Teton National Park. The magnitudes of this variation suggest to us that it may favor habitat selection within ponds and would lead to large differences in growth rates across ponds. The temperature differences can influence both growth rate and thus resource demand (as an example Pseudacris tadpoles hatch at 1 or $2 \mathrm{mg}$ and reach 250 to $300 \mathrm{mg}$ in 5 weeks of growth, a 150 to 
300 fold increase in demand per tadpole), and resource level itself. Tadpoles and snails fed throughout the water column during the day, but concentrate feeding at the water surface in the early morning. This diel pattern could represent habitat selection for higher temperatures, as the surface will warm more quickly than deeper waters when exposed to the sun. Whether the large temperature differences across and within ponds influences the outcome of species interactions is not clear, what is clear is that the variation in temperature is great enough that it may.

There is some evidence that differences in prey body sizes can influence the predation rate. Salamander larvae ate more of smaller tadpoles relative to larger tadpoles. Dragonfly naiads were apparently too small relative to larger tadpoles. Had the tadpoles been younger (and thus smaller), prey size might have been important to the dragonfly naiads. Finally, the dyticid larvae were so large relative to both larger and smaller tadpoles that they ate both. A smaller predator, or larger prey and the relative sizes of the prey might again influence the probability of predation. We did not examine the effect of body size on competitive interactions. Prior work has documented that relative body size can influence interference competition over food for tadpoles (Woodward 1987) or for froglets (Woodward 1986).

Our snail $\mathrm{x}$ tadpole experiment suggests that Pseudacris tadpoles and the snails are strong competitors. Snails reduced froglet weights by $23 \%$ while tadpoles reduced snail weight by $17 \%$. Drops of these magnitudes are likely to have strong negative consequences for both predation on these taxa (making it easier for the predators to capture the prey, and making the prey susceptible to predation for longer periods of time), and competition with other taxa (make them inferior competitors).

Predation and competition have strong interconnections. Competition occurs when resource demand outstrips supply. Predator-prey interactions lower prey densities, reducing the potential for competition to take place (Wilbur 1984, Woodward and Mitchell 1991). Competition reduces prey growth rates and thus makes the prey susceptible to size-limited predators for a greater period of time. Temperature, through an effect on growth rates has a large potential to influence competitive interactions, predator-prey interactions, and the interconnection between the two. We anticipate a lot more work examining the influence of temperature variation on species interactions in the montane pond system.

\section{$\downarrow \quad$ Literature Cited}

Morin, P. J. 1988. Salamander predation, prey facilitation, and seasonal succession in microcrustacean communities. pp. 174-187 In: Kerfoot, W. C. and A. Sih, eds. Predation: Direct and indirect impacts on aquatic communities. Univ. of New England Press, Hanover, England.

Wilbur, H. M. 1984. Complex life cycles and community organization in amphibians. In: Price, P. W., C. N. Slobodchikoff, W. S. Gaud, eds. A new ecology: novel approaches to interactive systems. Wiley, NY USA.

Woodward, B. 1983a. Tadpole size and predation in the Chihuahuan Desert. The Southwestern Naturalist 28:470-471.

Woodward, B. 1983b. Predator-prey interactions and breeding pond use by temporary pond species in a desert anuran community. Ecology 64:1549-1555.

Woodward, B. 1986. Paternal effects on juvenile growth in Scaphiopus multiplicatus (The New Mexican spadefoot toad). The American Naturalist 128:58-65.

Woodward, B. 1987. Tadpole interactions and breeding season duration of Woodhouse's toad (Bufo woodhousii). Copeia 1987:380386.

Woodward, B. and S. Mitchell. 1991. The ecology of desert amphibian communities. Chapter 9 in the Ecology of Desert Communities. G. Polis, ed., Univ. of Arizona Press. 